\title{
Sur un cas d'infestation du Copépode Eurytemora velox (Lillj) par une levure du genre Metschnikowia (Kamienski)
}

\author{
par A. FIZE, J.-F. MANIER et J. MAURAND \\ Laboratoire de Zoologie I ( $\mathrm{P}^{\mathrm{r}} \mathrm{O}$. TUZET), Faculté des Sciences, F 34 -Montpellier
}

\begin{abstract}
Résumé
La présence de Metschnikowia sp., levure ascosporée, est signalée comme parasite du Copépode Eurytemora velox en Camargue (France). Des observations sont faites sur les stades végétatifs, les asques fusiformes allongés ne contenant qu'une seule spore effilée aux deux extrémités. Le champignon envahit le corps du Crustacé entraînant sa mort.
\end{abstract}

\section{Summary}

The presence of Metschnikowia sp., ascosporous yeast is observed as parasitic in Copepod Eurytemora velox at Camargue (France). Observations on the vegetative stades, spindle-shaped lenghtened asci containing one only spore with two slender ends. The fungus fills the Crustacea's body and kills it.

En avril 1969, nous avons constaté qu'une levure du genre Metschnikowia (Metschnikoff) Kamienski (1) parasitait d'une façon intense une population du Copépode Eurytemora velox (Lillj.) (2). Le genre Metschnikowia comprend les espèces de levures

(1) Une étude exhaustive du genre a été établie par Miller et Van Uden, 1970 dans * The Yeasts (J. Lodder ed.). Nous remercions vivement le $\mathrm{D}^{r}$ Van Uden pour nous avoir transmis une copie du manuscrit de ce travail.

(2) Nous remercions le Professeur A. Raibaut de nous avoir donné ce matériel. E. velox qui appartient à la sous classe des Calanoida et à la famille des Temoridae, a été récolté en Camargue dans les eaux de l'Etang de l'Impérial, très exactement au lieu dit «Trabas du Rousty » à 5 km à l'est du village des Saintes-Maries-de-la-Mer (Gard).

Annales de Parasitologie humaine et comparée (Paris), t. 45, 1970, $\mathrm{n}^{\circ} 3$ 
caractérisées par la formation d'asques allongés ne contenant chacun qu'une ou deux spores en forme d'aiguille. La taxonomie de ce genre est assez complexe ; Miller et Van Uden (1970) en donnent une mise au point détaillée pour chaque espèce. Nous ne citerons ici que pour mémoire les synonymes les plus utilisés qui sont Monospora Metschnikoff 1884, Metschnikowiella Genkel 1913, Monosporella Keilin 1920 et Chlamydozyma Wickerham 1964. Quant aux Metschnikovellidae Caullery et Mesnil 1919, ainsi nommées parce qu'elles étaient considérées comme des levures apparentées aux Monospora de Metschnikoff, ce sont en réalité des microsporidies comme l'a révélé une étude au microscope électronique (Vivier, 1965).

C'est Metschnikoff (1884) qui décrit la première espèce du genre parasite de Daphnia magna à Odessa sous le nom de Monospora bicuspidata. Ce travail a un aspect historique car c'est en observant le processus d'infestation de la Daphnie par cette levure que Metschnikoff découvrit la phagocytose. D'après lui une forme semblable aurait été vue par Butchli (1876) dans le coelome du Nématode libre Tylenchus pellucidus Bast. Kamienski (1899) observe une levure similaire pathogène pour des Artemia salina de Mer Noire. Cest lui qui remplace le nom de Monospora déjà donné à une algue par celui de Metschnikowia qui est maintenant adopté. Différents zoologistes semblent avoir rencontré ce genre de levure comme parasites d'Invertébrés. Chatton (1907) mentionne qu'il trouve des Daphnies parasitées dans un bassin du Jardin des Plantes à Paris, chaque année au printemps. Caullery et Mesnil (1899) la signalent chez l'Annélide Polychète Potamilla toreili en Mer du Nord, deux ans plus tard ils (Mesnil et Caullery, 1911) étudient les tumeurs que ce parasite associé à une Haplosporidie détermine au niveau de certains segments de l'Annélide; ils auraient vu également une levure similaire chez le Copépode pélagique Acartia. Keilin (1920) décrit avec plus de détails Monosporella (= Metschnikowia) unicuspidata parasite de la cavité générale d'une larve de Diptère Cératopogonide (Dasyhelea obscura Winn.) en Angleterre.

Mais les levures sont à l'heure actuelle caractérisées par leurs propriétés physiologiques de fermentation et d'assimilation qui ne peuvent être connues qu'à partir de cultures pures; et c'est à des études récentes de microbiologistes que nous devons les importantes données nouvelles sur ces parasites. Van Uden et Castelo Branco (1961) cultivent pour la première fois deux espèces nouvelles Metschnikowia krissii et $M$. zobellii qu'ils isolent à partir d'eau de mer, de contenus de tubes digestifs de Poissons et de morceaux d'algues prélevées sur les côtes de Californie (La Jolla); ces deux espèces séparées par leurs propriétés physiologiques, malgré leur origine marine ont la possibilité de décimer rapidement des populations de Daphnia magna d'eau douce en produisant des asques voisins morphologiquement de ceux figurés par Metchnikoff. Les références concernant par la suite des Metschnikowia parasites d'Invertébrés sont peu nombreuses. Spencer, Phaff et Gardner, 1964 décrivent une Metschnikowia artemiae, ectoparasite d'Artemia salina récoltée dans un lac salé du Canada. Wickerham (1964) signale au'il a isolé depuis 1960 une Metschnikowia trouvée dans des sporocystes du Trématode Diplostomum flexicaudum parasite de l'hépato-pancréas d'une Limnée du lac Michigan. Enfin, Seki et Fulton (1969) étudient l'infestation 

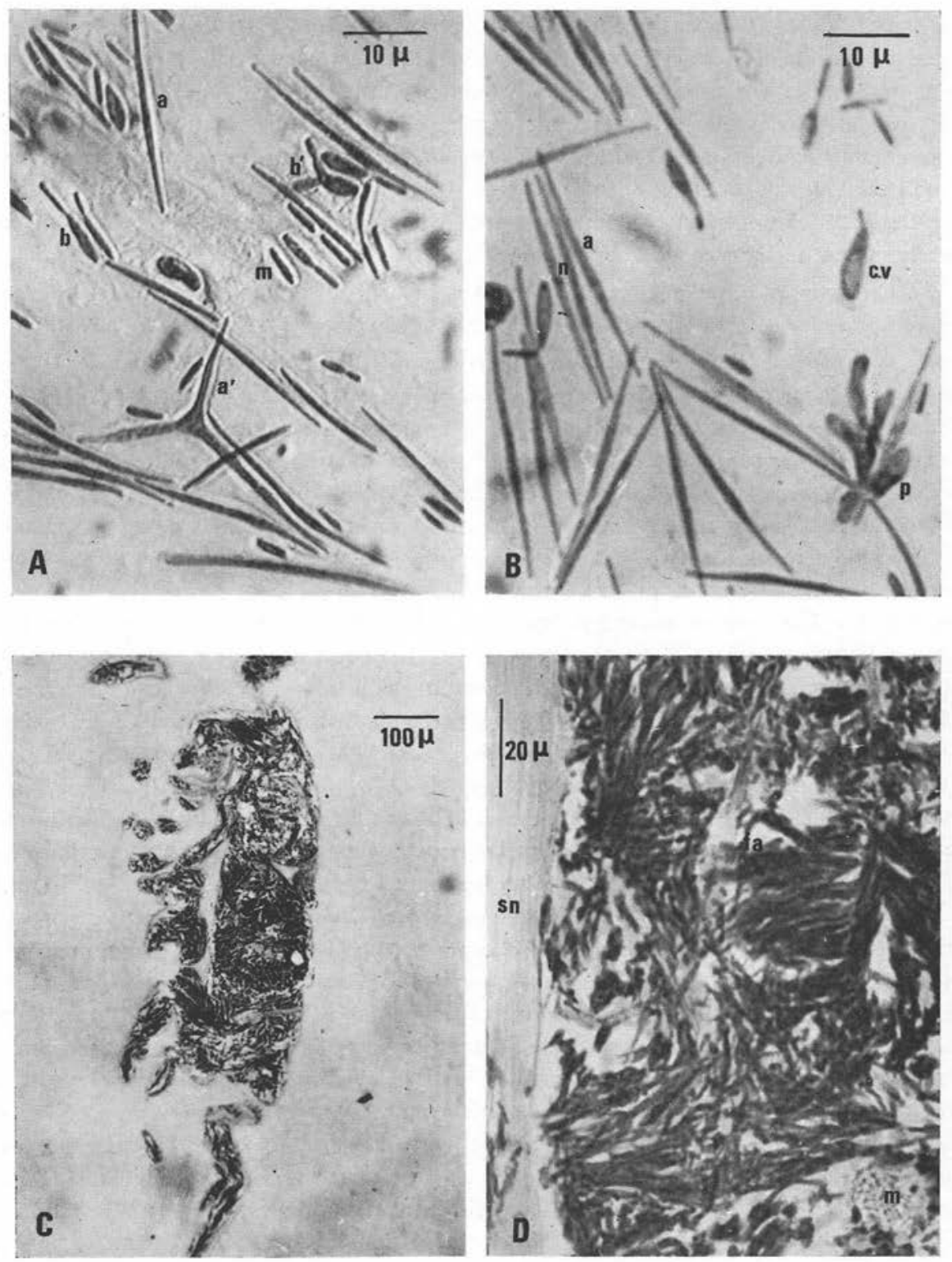

FIG. 1. - Metschnikowia sp. parasite d'Eurytemora velox (Lillj). A. a: asque typique; a': asque aberrant; $\mathrm{b}$ : cellule à un bourgeon; b' : cellule à deux bourgeons; $\mathrm{m}$ : cellule-mère. B. a : asque; cv: cellule vésiculeuse; $\mathrm{n}$ : noyau; $\mathrm{p}$ : pseudomycelium. C. coupe sagittale d'Eurytemora rempli de parasites. D. partie de la figure $\mathrm{C}$ grossie. $\mathrm{f}$ a: faisceau d'asques; $\mathrm{m}$ : muscle; s n : système nerveux. A. frottis Bouin A.P.S., contraste interférentiel ; B. frottis Bouin A.P.S. + Hématoxyline de Groat ; C. D. coupes Bouin A.P.S. 
par une Metschnikowia du Copépode Calanus plumchrus espèce prédominante du plancton dans le détroit de Géorgie (Pacifique). De ce dernier travail, on peut retenir que cette Metschnikowia serait plutôt d'origine terrestre avec des possibilités d'adaptation à la vie marine. Ses activités enzymatiques sont telles qu'elles peuvent être interprétées comme une adaptation au parasitisme. Les auteurs pensent que les nombreux exosquelettes vides de Calanus plumchrus récoltés en profondeur dans le détroit de Géorgie résultent certainement de l'action de ce parasite qui, de ce fait, a une incidence sur les quantités totales de zooplancton récolté.

Nos propres observations ont été faites à partir d'une population de Copépodes vivants, de deux séries de coupes et de frottis diversement colorés.

Le Copépode Eurytemora velox (Lillj.) est un Calanoïde euryhalin d'asséz grande taille dont la présence en Camargue a été signalée pour la première fois par Petit et Schachter (1947). Plus ou moins incolore, il prend un aspect blanc laiteux lorsqu'il est parasité. Il est à ce stade déjà très envahi par les différentes formations de la levure faciles à observer sur le vivant après dilacération du corps de l'hôte. Les asques prédominent et forment un feutrage de fuseaux à renflement submédian d'une trentaine de $\mu$ de long, incolores mais très réfringents (fig. 2, A). Nos observations sur le vivant ont été malheureusement un peu trop rapides car nos recherches à l'époque de la récolte des Eurytemora portaient sur l'étude de Microsporidies, qui donnent elles aussi à l'hôte un aspect blanc laiteux. Il aurait fallu faire une étude d'une population complète des Eurytemora de la localité pour avoir une meilleure connaissance de l'évolution des formes de la levure au cours de l'infestation et surtout à ses débuts. Les asques et les autres formations de la levure sont visibles sur différents frottis. Les ébauches de pseudomycelium sont rares (fig. $1 \mathrm{~B}, \mathrm{p}$ ). Des cellules très vésiculeuses avec une seule grosse vacuole s'en détachent (fig. $1 \mathrm{~B}, \mathrm{cv}$ ); ces cellules isolées forment le plus souvent un bourgeon unique qui semble évoluer en une cellule cylindrique allongée dont les plus courtes ont $5 \mu$ de long sur 1,6 $\mu$ de large (fig. $1 \mathrm{~A}, \mathrm{~m}$ ). Traités à l'A.P.S. et à l'hématoxyline de Groat, les asques apparaissent fortement colorés en rouge violacé avec une masse nucléaire centrale (fig. $1 \mathrm{~B}, \mathrm{n}$ ); colorés uniquement à l'hématoxyline de Groat, les éléments montrent un cytoplasme basophile entourant les vacuoles incolores; au Feulgen-Vert-Lumière, la masse nucléaire est rose tandis que l'asque reste incolore. La spore individualisée dans l'asque révèle un corps central peu visible, se prolonge par deux pointes fines d'inégales longueurs, d'aspect vrillé et très réfringentes (fig. 2, C, D, flèches). Des coupes examinées au microscope électronique nous ont nettement prouvé que cette spore était unique dans l'asque et présentait une ornementation de stries très fines à disposition légèrement hélicoïdale. Signalons que nous avons exceptionnellement rencontré des asques en forme d'Y (fig. $1 \mathrm{~A}$, a') : ils semblent provenir d'un bourgeonnement double d'une cellule-mère (fig. $1 \mathrm{~A}$, b'). Nous pouvons, enfin, peut-être interpréter comme une conjugaison les images de la figure 2, B (flèches).

Les coupes sagittales nous montrent que le parasite envahit la cavité générale (fig. 1, C). Sur l'une de nos séries de coupes, les différents organes digestifs, nerveux et génitaux sont encore visibles. Sur une autre série, seuls le système nerveux et les 

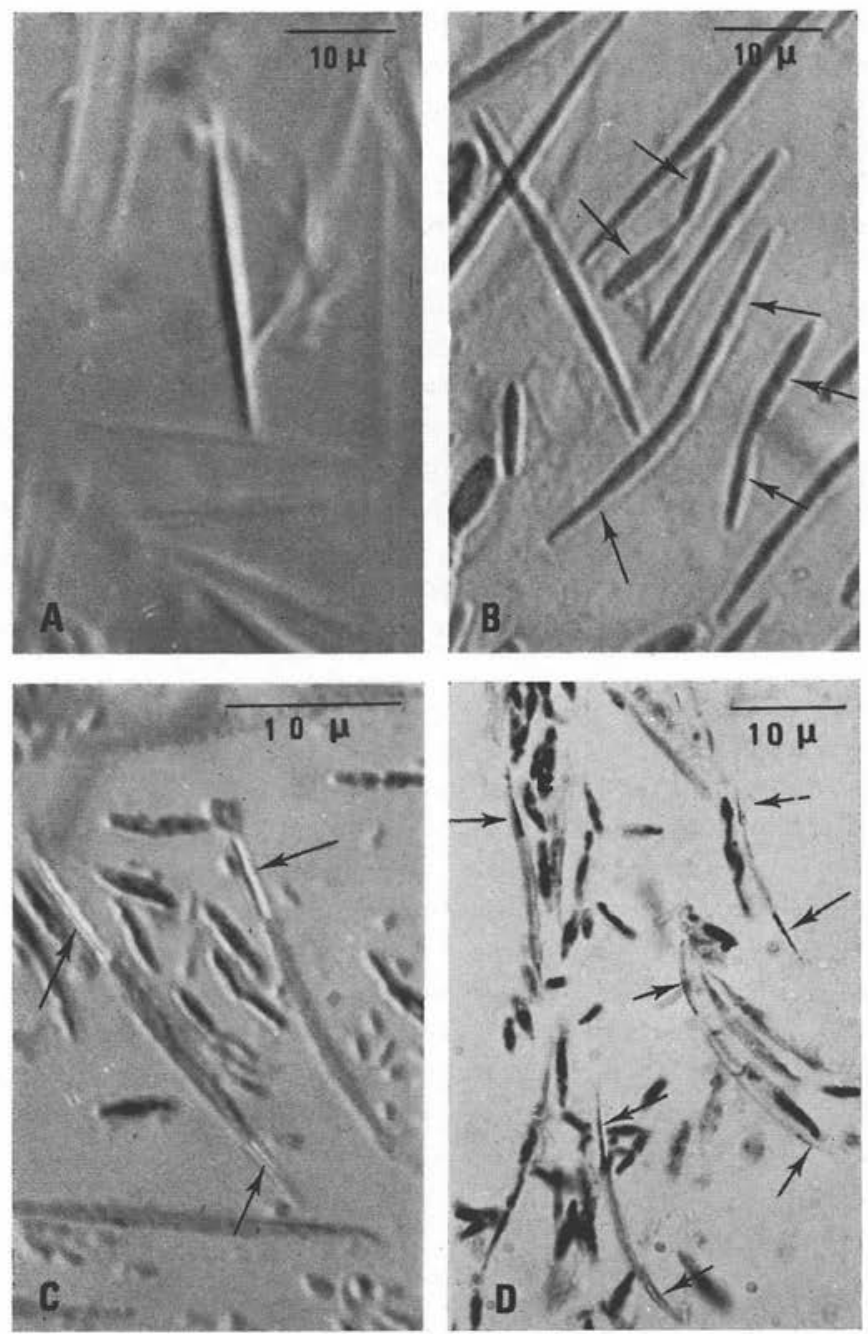

Fig. 2. - Metschnikowia sp. A. asque; B. conjugaison présumée de deux cellules (flèches). C. et D. asques enveloppant une ascospore ayant à chaque pôle une ornementation aciculaire (flèches). A. frottis sur le vivant, contraste interférentiel; B. frottis Bouin A.P.S., contraste interférentiel; C. frottis Bouin hématoxyline de Groat, contraste interférentiel; D. frottis Bouin hématoxyline de Groat

muscles subsistent (fig. 1, D). Les asques qui constituent la phase ultime du développement arrivent à être serrés les uns contre les autres et vont envahir même les plus fines soies. 
Nous n'avons pas suivi le cycle complet de cette levure mais tout laisse supposer qu'il s'effectue conformément à la description donnée par Metschnikoff chez la Daphnie. L'hôte bourré d'asques meurt, un autre Copépode ingère des asques; la spore est libérée dans le tube digestif du nouvel hôte dont elle traverse l'épithélium intestinal. Elle germe dans la cavité générale et redonne les cellules-mères. A cette action mécanique de pénétration de la spore, il faut ajouter une action enzymatique de la levure sur certaines subśtances organiques de l'animal parasité. C'est au niveau du passage de la spore dans la cavité générale que Mestchnikoff observa le phénomène de la phagocytose. Les leucocytes de la Daphnie se rassemblent autour de la spore en formant un plasmode qui l'englobe et la digère. Les spécimens d'Eurytemora velox observés étaient tous dans un état de parasitisme avancé : c'est la raison pour laquelle nous n'avons observé aucune figure de germination des spores et n'avons eu qu'une seule image nette de phagocytose de cellules-mères par une cellule indéterminée de l'hôte.

Nous ne pouvons évidemment prétendre à situer notre espèce par rapport aux cinq espèces définies à l'heure actuelle car nous ne connaissons pas ses propriétés physiologiques.

Cependant sa caractéristique de posséder une spore unique comme nous l'a montré la microscopie électronique l'écarte de $M$. bicuspidata (Metschnikoff) Kamienski et de ses variétés qui possèdent deux spores par asque (Miller, Barker et Pitt 1967, Miller et Van Uden 1970). Seules $M$. krissii et $M$. zobelii possèdent une spore unique dans l'asque, mais cette spore n'est pointue qu'à une seule extrémité, ce qui ne semble pas le cas pour l'espèce parasite d'Eurytemora.

L'étude de cette levure trouvée chez Eurytemora velox en Camargue demanderait évidemment à être reprise avec les nouvelles méthodes d'investigation. Il nous a tout de même paru intéressant de signaler la présence dans les eaux saumâtres du midi de la France de ce champignon pathogène: il décime des populations d'Eurytemora velox et de ce fait pourrait avoir une influence sur les chaînes alimentaires dans les étangs du Bas-Rhône-Languedoc.

\section{Bibliographie}

Ainsworth (G. C.), 1961. - Dictionary of the fungi. Common mycol., Inst., Kew, Surrey, $547 \mathrm{p}$.

BüTsChli (O.), 1876. - Studien über die ersten Entwicklungsvorgänge der Eizelle, die Zelltheilung und die Conjugation der Infusorien. Abhandl. d. Senckenb, naturf. Gesselsch., 10, p. 148, Pl. XIV, fig. 8.

Caullery (M.) et Mesnil (F.), 1899. - Sur les parasites internes des Annélides Polychètes en particulier de celles de la Manche. C.R. $28^{\circ}$ Session Ass. Fr. Avanc. Sci., Boulognesur-Mer, p. 491-496.

—, —, 1919. - Metschnikovellidae et autres protistes parasites des Grégarines d'Annélides. Ann. Inst. Pasteur, Paris, 33, p. 209-240. 
Chatton (E.), 1907. - Revue des parasites et commensaux des Cladocères. Observations sur des formes nouvelles ou peu connues. C.R. Ass. Fr. Avanc. Sci., Congrès de Reims, p. 797-811.

GenKel (A.), 1913. - Essay on sporulating plants. Moscow : Knebel.

KAMIENSKI (T.), 1899. - Notice préliminaire sur la nouvelle espèce de Metschnikowia (Monospora Metsch.). Trav. Soc. Imp. Natural. S. Petersb., 30, p. 363-364 (en russe).

KeIlen (D.), 1920. - On a new Saccharomycete Monosporella unicuspidata gen. n. nom. n. sp. parasitic in the body cavity of a dipterous larva (Dasyhelea obscura Winnertz). Parasitology, 12, p. 83-91.

Mesnil (F.), et Caullery (M.), 1911. - Néoformations papillomateuses chez une Annélide (Potamilla torelli) dues probablement à l'influence de parasites (Haplosporidie et levure). Bull. biol. Fr. et Belg., 45, p. 89-105.

Metschnikoff (E.), 1884. - Ueber eine Sprosspilzkranheit der Daphnien. Beitrag zur Lehre über den Kampf der Phagocyten gegen Krankheitserreger. Arch. f. pathol. Anat. und Physiol., 96, p. 177-195, Pl. IX, X.

Miller (M. W.), BarKer (E. R.) and Pitt (J. I.), 1967. - Ascospore numbers in Metschnikowia. J. Bact. U.S.A., 94, p. 258-259.

- and van Uden (N.), 1970. - Genus Metschnikowia dans The Yeasts. J. Lodder ed., North Holland Publishing Co.

Petit (G.) et Schachter (D.), 1947. - Sur la présence du genre Eurytemora en Camargue. Bull. Mus. Hist. Nat. Marseille, 7, $\mathrm{n}^{\circ}$ 2-3, p. 103-114.

SEKI (H.) and Fulton (J.), 1969. - Infection of marine copepods by Metschnikowia sp. Mycopathol. Mycol. appliquée, Pays-Bas, 38, 2, p. 61-70.

SPencer (J. F. T.), Phaff (H. J.) and Gardner (N. R.), 1964. - Metschnikowia kamienskii sp. n., a yeast associated with brine sphrimp. J. Bact., 88, p. 758-762.

Uden (N. van), 1962. - On the nomenclature of the genus Metschnikowia Kamienski. Rev. Biol., 3, p. 95-96.

- and Castelo Branco (R.), 1961. - Metschnikowiella zobelii sp. nov. and M. krissii sp. nov. two yeasts from the Pacific Ocean pathogenic for Daphnia magna. J. gen. Microbiol., 26, p. 141-148.

- and Fell (J. W.), 1968. - Marine yeasts in « Advances in Microbiology of the sea by Droop M. R. and Ferguson Wood E. J.». Academic Press, London and New York, p. $167-201$.

VIVIER (E.), 1965. - Etude, au microscope électronique, de la spore de Metschnikovella hovassei n.sp.; appartenance des Metschnikovellidae aux Microsporidies. C.R. Acad., Sci. Paris, 260 , p. $6982-84$.

Wickerham (L. J.), 1964. - A preliminary report on a perfect family of exclusively protosexual yeast. Mycologia, 56, p. 253-266. 